Published in final edited form as:

J Acoust Soc Am. 2008 February ; 123(2): 998-1007.

\title{
Gap detection in modulated noise: Across-frequency facilitation and interference
}

\author{
John H. Grose, Emily Buss, and Joseph W. Hall III \\ Dept. Otolaryngology - Head \& Neck Surgery, University of North Carolina at Chapel Hill, Chapel \\ Hill, NC 27599-7070
}

\begin{abstract}
This study tested the hypothesis that a detection advantage for gaps in comodulated noise relative to random noise can be demonstrated in conditions of continuous noise and salient envelope fluctuations. Experiment 1 used five $25-\mathrm{Hz}$ wide bands of Gaussian noise, low-fluctuation noise, and a noise with increased salience of the inherent fluctuations (staccato noise). The bands were centered at $444,667,1000,1500$, and $2250 \mathrm{~Hz}$, with the gap signal always inserted in the 1000- $\mathrm{Hz}$ band. Results indicated that a gap detection advantage existed in continuous comodulated noise only for Gaussian and staccato noise. Experiment 2 demonstrated that the advantage did not exist for gated presentation. This experiment also showed that the advantage bore some similarity to comodulation masking release (CMR). However, differences were also noted in terms of the effects of the number of flanking bands and the absence of a detection advantage in gated conditions. The detrimental effect of a gated flanking band was less pronounced for a comodulated band than for a random band. This study indicates that, under some conditions, a detection advantage for gaps carried by a narrow band of noise can occur in the presence of comodulated flanking bands of noise.
\end{abstract}

\section{INTRODUCTION}

The detection of a temporal gap carried by only one of the components in a complex stimulus is typically more difficult than if many of the components carry the gap synchronously. For example, Green and Forrest (1989) found that sensitivity to a gap imposed upon a single tone in a 21-tone complex was poorer than if the gap was imposed upon 11 of the 21 tones simultaneously. In a similar vein, Hall et al. (2007) observed that sensitivity to a gap carried by one $50-\mathrm{Hz}$-wide narrow band of noise in a concurrent complex of four such bands was poorer than if all four bands carried the gap synchronously. Other work has used as a baseline the detectability of a gap in a single noise band presented in isolation. Relative to this baseline, gap detection performance typically improves if additional noise bands are added that also contain the synchronous gap (Grose and Hall, 1988; Grose, 1991; Hall et al., 1996; Grose and Hall, 1997). At the same time, gap detection performance typically declines if additional noise bands are added that do not contain the gap (Grose and Hall, 1993a; Moore et al., 1993).

Although some of this work has focused on the manner in which temporal cues are combined across frequency (e.g., Grose and Hall, 1997; Hall et al., 2007), other work has focused on the modulation characteristics of the multiple noise bands comprising the stimulus complex. This interest stems in part from the phenomenon of comodulation masking release (CMR), wherein a detection advantage is conferred upon a signal masked by a narrow band of noise when additional bands of noise are present that share the same modulation pattern as the on-signal masker (for review, see Grose et al., 2005a). One interpretation of CMR has been that the presence of the comodulated flanking bands allows for a detection process based upon 
sensitivity to across-frequency envelope decorrelation. If this interpretation is correct, it follows that such a process might confer a detection advantage on gaps in comodulated noise, relative to random noise, as well as tones. That is, if a gap is imposed on one of several otherwise comodulated bands of noise, then an across-frequency envelope decorrelation would occur for the duration of the gap, including the transition ramps. Sensitivity to this decorrelation could signal the presence of the gap. This hypothesis was tested by Grose and Hall (1993a) using a single flanking band of noise, and by Moore et al. (1993) using eight flanking bands of noise. Both studies found that the presence of additional bands of noise not carrying the gap - either comodulated or random - was detrimental to detection of a gap in a single target band of noise. This finding is not consistent with predictions based on CMR findings. The general conclusion was that the across-frequency effects observed were more akin to modulation detection interference (e.g. Yost and Sheft, 1989; Moore and Jorasz, 1992), where the presence of amplitude modulation at a remote frequency degrades sensitivity to temporal features of the target stimulus envelope. Although it might be argued that the signal duration in the CMR paradigm is longer than the typical duration of temporal gaps at threshold, this duration difference is unlikely to account for the failure to observe a gap detection benefit in the presence of comodulated flanking bands: CMR has been observed for signal durations as short as $25 \mathrm{~ms}$ (Schooneveldt and Moore, 1989a), and thresholds for detecting gaps in narrow bands of noise can be over twice as long as this (e.g. Grose et al., 1989).

The purpose of the present investigation was to reassess the generality of the conclusion that comodulated flanking bands do not facilitate the detection of a gap presented in a target band. The study was motivated by two converging lines of thought. The first line was the consideration that all of the gap detection studies noted above used gated stimuli. In typical gap detection studies, the stimuli are gated on for the duration of each observation interval and, in the target observation interval, a gap is imposed at some point in the waveform. The magnitude of CMR is dependent upon the gating characteristics of the comodulated noise bands - at least when the number of bands is relatively few - such that CMR is greater when the comodulated noise bands are presented continuously than when the bands are gated on and off synchronously during the observation intervals (Hatch et al., 1995). Pilot listening comparing gap detection in similar conditions of gated vs. continuous noise indicated that a detection advantage appeared to be present for gaps in the presence of comodulated flanking bands for the continuous mode of presentation. The second converging line was the notion that the limiting factor in the sensitivity to gaps carried by a narrow band of noise is the perceptual similarity between the imposed gap and the on-going fluctuations inherent to the noise band, as expanded on below. If the detectability of the gap is limited by confusion with the inherent random dips in the noise, then the presence of comodulated flanking bands might disambiguate the imposed gap from the on-going fluctuations. A formal experiment was therefore undertaken to test the hypothesis that gap detection benefits from the presence of comodulated flanking bands under conditions of perceptual confusion between imposed gap and inherent fluctuation, and that the benefit is more likely to occur in continuous, rather than gated, modes of presentation.

As just noted, a necessary premise of this hypothesis is that gap threshold in the case of the signal band alone must be limited by factors other than the absolute temporal acuity of the auditory system. That is, any reduction in gap detection threshold brought about by the addition of comodulated flanking bands must imply that temporal processing is not operating at the limits of acuity in the single-band case. It has long been recognized that detection of a gap in a narrow band of noise is likely limited by a perceptual confusion between the imposed gap and the on-going, perceptually salient, fluctuations inherent to narrow bands of noise (Shailer and Moore, 1983; Eddins et al., 1992). If so, it follows that manipulation of the magnitude of the confusion effect should affect the degree of benefit conferred by the presence of the comodulated flanking bands. In a study of the effect of envelope fluctuations on gap detection, 
Glasberg and Moore (1992) demonstrated that, as the degree of envelope fluctuation of a narrow band of noise is systematically varied by raising the envelope to powers greater or less than unity, the detectability of a gap imposed in the noise band correspondingly varies. This supports the notion that variation in sensitivity to the gap reflects the perceptual salience of the ongoing fluctuations in the noise and, hence, the magnitude of the confusion effect. In the present study, the magnitude of the confusion effect was also manipulated by varying the salience of the on-going envelope fluctuations.

In summary, the purpose of this study was to test the hypothesis that a detection advantage for gaps in comodulated noise can be demonstrated in conditions of continuous noise and perceptually salient envelope fluctuations. Two main experiments were undertaken, and one supplementary control experiment. Experiment 1 employed continuous presentation of noise stimuli with differing properties of envelope fluctuation. Experiment 2 focused on gated noise presentation, but included some continuous conditions designed to test whether observed effects could be attributed to CMR-like mechanisms. The supplementary experiment tested a subset of gated conditions analogous to those used in experiment 2 but employed gap characteristics more similar to those of previous studies.

\section{EXPERIMENT 1. GAP DETECTION IN CONTINUOUS NOISE BANDS}

\section{A. Method}

Observers-Six normal-hearing observers participated in experiment 1, ranging in age from 26 to 56 years (mean $=45$ years). All had audiometric thresholds $\leq 20 \mathrm{~dB} \mathrm{HL}$ at the octave frequencies $250-8000 \mathrm{~Hz}$ (ANSI, 1996). All were experienced observers in psychophysical tasks, including temporal processing tasks.

Stimuli-The stimuli were $25-\mathrm{Hz}$ wide bands of noise centered at 444, 667, 1000, 1500, and $2250 \mathrm{~Hz}$; these frequencies are equally spaced on a logarithmic scale. The $1000-\mathrm{Hz}$ band was the signal band in all cases. In the baseline condition this band was presented in isolation. In the remaining conditions, either a single flanking band was present - always centered at 1500 $\mathrm{Hz}$ - or all four flanking bands were present. When present, the flanking bands either had the same temporal envelope as the signal band, or all bands had independent envelopes. In all cases the gap was introduced by gating the $1000-\mathrm{Hz}$ signal band off and on with a half cycle of a raised-cosine function, $42 \mathrm{~ms}$ in duration. The gap duration was quantified as the time between the initiation of gate offset and the initiation of gate onset. With this metric, for example, a 42ms gap would be generated by initiating the onset ramp immediately after completion of the 42-ms offset ramp. The 42-ms ramps were chosen in part to minimize spectral splatter associated with the introduction of the gap, but also to avoid a perceptual distinction between the imposed gap and the inherent fluctuations of the signal noise band. Given the approximately $16-\mathrm{Hz}$ average fluctuation rate associated with a $25-\mathrm{Hz}$ wide Gaussian noise (Rice, 1954), it was desirable to use a ramp time that was not shorter than half the 62-ms period. The notion was that, by avoiding a sharp perceptual contrast between the imposed gap and the inherent fluctuations of the noise, the confusion effect would be heightened by a 'blending' of the gap with the on-going fluctuations.

Three sets of comodulated and random noise bands were generated that differed with respect to their modulation characteristics. The first set consisted of bands of Gaussian noise (GN), the second set consisted of bands of low-fluctuation noise (LFN), and the third set consisted of bands of staccato - or 'choppy' - noise (SN). Exemplars of each type of noise are shown in Fig. 1 (upper panel: waveforms, lower panel: distribution of the instantaneous amplitudes). Each random GN band was constructed by generating a Gaussian noise sample and filtering it in the frequency domain by means of multiplication with a pair of boxcar functions placed symmetrically around the Nyquist frequency. To generate the comodulated GN bands, the 
1000-Hz signal band was first generated, and then the real and imaginary components associated with this band were replicated at the spectral locations of the other four bands. This procedure results in noise bands with perfectly correlated envelopes across frequency. The LFN bands were generated following a procedure outlined by Kohlrausch et al. (1997, Method 1). Briefly, a Gaussian noise signal band was generated as described above. The Hilbert envelope was then calculated and the time-domain stimulus subsequently divided by this envelope on a point-by-point basis. This modified time-domain waveform was then transformed into the frequency domain, refiltered to the original $25-\mathrm{Hz}$ passband, and transformed back into the time domain. This entire procedure was repeated eight times, resulting in a $25-\mathrm{Hz}$ wide noise band with a markedly reduced crest factor. For random LFN bands, the generation process was repeated independently for each of the four flanking bands. For comodulated LFN bands, the real and imaginary components characterizing the final rendition of the signal band were used to generate the flanking bands. The SN bands were generated using procedures analogous to those used for the LFN bands. First, a band of Gaussian noise was generated as described above and the Hilbert envelope calculated. This envelope was used to create a target envelope comprised of zeros and ones: the target envelope was set to zero for each point in the Hilbert envelope that fell at or below 0.8 of the mean Hilbert envelope value, and the target envelope was set to one for each point above this criterion. The original GN band was multiplied by this target envelope and then refiltered in the frequency domain to the original $25-\mathrm{Hz}$ bandwidth. This process was repeated 20 times to result in a $25-\mathrm{Hz}$ wide band of noise that had an accentuated interrupted quality. Random and comodulated flanking bands for the SN set were generated in the same manner as described for the GN and LFN sets.

All stimuli were generated in MATLAB (Mathworks) and stored on disk as a waveform library. Each stimulus array was $2^{18}$ points long, which corresponds to a 21.5 second sample when played out at a digital-to-analog conversion rate of $12,207 \mathrm{~Hz}$. Because stimuli were converted from the frequency to the time domain via an inverse Fourier transform, the noise band waveforms could be output cyclically and continuously with seamless repetition. Gating was applied in software (RPvds, Tucker-Davis Technologies [TDT]). Stimuli were played out of a real-time DSP processor (RP2, TDT) at a level per noise band of $55 \mathrm{~dB}$ SPL, similar to the level used in Grose and Hall (1993a). All stimuli were presented to the left earphone of a Sennheiser HD265 Linear headset.

Procedure-A 3-alternative forced-choice (3AFC) paradigm was employed, with each of the 500-ms listening intervals separated by $450 \mathrm{~ms}$. To prevent a gap from exceeding the 500ms listening interval, a ceiling value of $499 \mathrm{~ms}$ was imposed on the threshold estimation procedures. The gap was temporally centered in one of the three intervals, selected at random. Thresholds were estimated using a 3-down, 1-up adaptive track that converged on the 79\% correct point on the psychometric function. Initially, gap durations were adjusted by a factor of 1.41. This was reduced to 1.19 after the second track reversal. The track continued until 8 reversals had been obtained, and the threshold estimate for a track was computed as the geometric mean of the gap duration at the last 6 track reversals. Observers indicated their responses by means of a handheld response box. Listening intervals were marked by boxmounted LEDs, and correct interval feedback was provided by the same LED markers.

Each observer participated in five conditions for each of the three noise band sets, for a total of 15 conditions. One condition in each set was a baseline condition using the 1000-Hz signal band presented alone. Two conditions in each set used the signal band plus a single, $1500-\mathrm{Hz}$, flanking band; in one condition the flanking band was comodulated with the signal band, while in the other condition it was independent. The single flanking band used by Grose and Hall (1993a) was also centered at $1500 \mathrm{~Hz}$. The final two conditions in each set used the signal band plus all four flanking bands; again, in one of these conditions all bands were comodulated, 
whereas in the other condition each band was independent of all the others. For each observer and condition, at least three estimates of gap threshold - but often four or five, if time permitted - were measured. The observer's final threshold for each condition was taken as the geometric mean of all estimates collected for that condition. Conditions were blocked, but the order of blocks was random across observers.

\section{B. Results and Discussion}

The results of experiment 1 are shown in Fig. 2. Three of the panels show the individual and group mean data for each of the three noise types separately; the fourth panel shows the group means for all three noise types overlaid (circles: SN, squares: GN, triangles: LFN). Three key data patterns are apparent: [1] for the two noise types with the most pronounced envelope fluctuations ( $\mathrm{SN}$ and GN), gap thresholds in the presence of comodulated flanking bands appear lower than when the signal band is presented in isolation (i.e., a detection advantage is evident); [2] for SN and GN, gap thresholds appear lower for comodulated flanking bands than random flanking bands (i.e., the detection advantage is specific to comodulated bands); and [3] neither of the above patterns is evident in the minimally fluctuating LFN. Before examining these patterns quantitatively, a key preliminary question is whether gap detection performance deteriorated as the degree, or salience, of the modulation increased. Recall that a premise of the experiment was that gap detection is limited by a perceptual confusion between the imposed interruption and the inherent fluctuation pattern of the stimulus such that the more salient the fluctuation pattern, the higher the gap detection threshold. Mean gap thresholds in the signal band alone condition for SN, GN and LFN were 111, 67, and $24 \mathrm{~ms}$, respectively. (For comparison, Grose et al. (1989) measured an average gap threshold of $70 \mathrm{~ms}$ for a (gated) 25Hz-wide Gaussian noise band, with the gap bounded by 33 -ms ramps.) Thresholds in the signal band alone condition were compared for the three types of noises by means of a repeated measures analysis of variance (RANOVA). Note that in this, and all analyses in this study, the $\log$ transforms of the data were used to ensure homogeneity of variance. The analysis revealed a significant effect of noise type $\left(\mathrm{F}_{2,10}=515.65 ; \mathrm{p}<0.01\right)$, and post-hoc contrasts indicated that thresholds for the GN band were significantly higher than for the LFN band and significantly lower than for the $\mathrm{SN}$ band $\left(\mathrm{F}_{1,5}=277.66 ; \mathrm{p}<0.01\right.$ and $\mathrm{F}_{1,5}=104.74 ; \mathrm{p}<0.01$, respectively). As expected, therefore, gap detection was best in the noise with minimal envelope fluctuation (LFN) and poorest in the noise with accentuated fluctuation (SN).

The prediction associated with the experimental hypothesis was that detection of the gap in the $1000-\mathrm{Hz}$ signal band would improve with the addition of comodulated flanking bands (sans gap) in those cases where performance in the single band case was limited, not by absolute temporal acuity per se, but by a perceptual confusion effect. To test this prediction, RANOVAs were performed on the data for each of the noise types separately to determine the effects of the flanking bands within each set. For the LFN set, the RANOVA indicated a significant effect of condition $\left(F_{4,20}=7.10 ; p<0.01\right)$, and post-hoc contrasts revealed that this effect was due to all conditions with flanking bands - both random and comodulated - yielding higher gap thresholds than the baseline signal band alone condition $\left(\mathrm{F}_{1,5}\right.$ ranging from $10.24-47.66 ; \mathrm{p}$ ranging from $0.001-0.024)$. Thus, for noise bands with minimal fluctuations, where gap detection performance in the baseline condition was relatively acute, the presence of comodulated flanking bands was disadvantageous to performance, as was the presence of random flanking bands. The finding that flanking bands, even with minimal fluctuations, can be disruptive to gap detection is in line with the study of Grose and Hall (1993a) who showed that, in gated conditions, detection of a gap in a narrow band of noise can be disrupted by the presence of a pure tone not carrying the gap.

For the GN set, a RANOVA indicated a significant effect of condition $\left(\mathrm{F}_{4,20}=25.16 ; \mathrm{p}<0.01\right)$, and post-hoc contrasts indicated that all conditions with flanking bands resulted in gap 
thresholds significantly different from baseline $\left(\mathrm{F}_{1,5}\right.$ ranging from $11.85-23.75$; $\mathrm{p}$ ranging from $0.005-0.018$ ). It is evident from Fig. 2 that these differences from baseline are due to the comodulated flanking band conditions yielding thresholds lower than baseline, whereas random flanking band conditions yielded thresholds higher than baseline. A RANOVA examining only the two factors associated with the flanking band conditions (modulation coherence and flanking band number) indicated a significant effect of modulation coherence $\left(F_{1,5}=89.75 ; p<0.01\right)$, but no effect of flanking band number $\left(F_{1,5}=1.40 ; p=0.29\right)$. However, the interaction between these two factors was significant $\left(\mathrm{F}_{1,5}=20.67 ; \mathrm{p}<0.01\right)$, indicating that the difference in gap threshold associated with comodulated $v s$. random flanking bands increased as the number of flanking bands increased. This pattern of results indicates that, for narrow bands of continuous Gaussian noise, gap detection performance improves in the presence of comodulated flanking bands and deteriorates in the presence of random flanking bands.

Finally, for the SN set, a RANOVA indicated a significant effect of condition $\left(\mathrm{F}_{4,20}=23.59\right.$; $\mathrm{p}<0.01$ ), but post-hoc contrasts indicated that only the conditions with comodulated flanking bands resulted in gap thresholds significantly different from baseline $\left(F_{1,5}=61.92 \& 44.71\right.$, respectively; $\mathrm{p}<0.01$ ). To assess the effects of modulation coherence and flanking band number, a RANOVA was performed on the conditions with flanking bands. The analysis indicated a significant effect of modulation coherence $\left(\mathrm{F}_{1,5}=69.69 ; \mathrm{p}<0.01\right)$, but no effect of flanking band number $(\mathrm{F} 1,5=1.49 ; \mathrm{p}=0.28)$. The interaction between these two factors was also not significant $\left(F_{1,5}=3.78 ; p=0.11\right)$. This pattern of results indicates that in the noise bands with accentuated fluctuation, the presence of comodulated flanking bands was advantageous to gap detection but performance was unaffected by the presence of random flanking bands.

The results of this experiment support the hypothesis that gap detection benefits from the presence of comodulated flanking bands under conditions of continuous presentation. However, this gap detection advantage is restricted to noise types with salient envelope fluctuations where performance is likely to be limited by a confusion effect between the imposed gap and the on-going fluctuations. In further support of this interpretation, it is interesting to note that the magnitude of the gap detection advantage in the presence of comodulated flanking bands was greater for the SN noise type than for the GN noise type. For example, the improvement in gap detection threshold when four comodulated bands were added to the signal band was $48.6 \mathrm{~ms}$ for the SN bands and $16.6 \mathrm{~ms}$ for the GN bands, a difference that is significant $\left(\mathrm{t}_{5}=4.71 ; \mathrm{p}<0.01\right)$.

The finding here that the presence of comodulated flanking bands can aid gap detection in continuous noise is in contrast with previous findings indicating no such effect for gated noise. It is possible that this dissimilarity is related to the difference in CMR magnitude seen for continuous $v s$. gated presentation in conditions where relatively few flanking bands are present. However, although the gap detection advantage observed here in the presence of comodulated flanking bands is, in some respects, analogous to CMR, the pattern of data is not consistent with CMR findings. Most studies of CMR show an asymptotic dependence of the magnitude of masking release on the number of flanking bands (Carlyon et al., 1989; Schooneveldt and Moore, 1989b; Haggard et al., 1990; Hall et al., 1990; Hatch et al., 1995). In the present study, where a gap detection advantage was evident, the advantage did not consistently depend on the number of comodulated flanking bands present. Furthermore, at a phenomenological level, the gap detection effect also appears qualitatively different from CMR: In CMR, the masked signal is generally perceived as continuous during its presentation, and the presence of comodulated flanking bands generally enhances the salience of the signal. In the gap detection task, the occurrence of the gap in the presence of the comodulated flanking bands was often described by observers as being signaled by its offset - i.e., by the onset of the signal band at 
the termination of the gap. In other words, the gap itself is not more salient; rather, the onset of the noise band carrying the gap became more prominent at the termination of the gap. (A typical description was that the signal band "pings on" at the conclusion of the gap.) This description is reminiscent of the auditory enhancement effect noted by Viemeister and Bacon (1982), and others. To examine the relation between comodulation and gap detection further, and to explore the relation between gap detection advantage and CMR, a second experiment was undertaken that used gated presentation and also included conditions designed to test for the presence of CMR-like behavior. Only the two types of noise that resulted in a gap detection advantage (GN and $\mathrm{SN}$ ) were used in experiment 2.

\section{EXPERIMENT 2. GAP DETECTION IN GATED NOISE BANDS}

Existing studies that have examined the detection of gaps in single noise bands in the presence of other noise bands - either comodulated or random - have found no indication of a detection advantage (Grose and Hall, 1993a; Moore et al., 1993; Hall et al., 2007). All of these studies used gated presentation. A goal of experiment 2 was to determine whether the detection advantage observed in experiment 1 was restricted to conditions that used a continuous presentation mode. A second goal was to determine whether the detection advantage associated with comodulated flanking bands in experiment 1 was diminished under conditions that have been shown to reduce CMR. Two stimulus manipulations were implemented to test for similarities with CMR.

The first manipulation - gating asynchronies among components - has been used in studies of CMR that have focused on auditory grouping effects (e.g., Grose and Hall, 1993b; Grose and Hall, 1996; Hall et al., 1997; Dau et al., 2004). Because auditory grouping behavior is generally disrupted by gating asynchronies among components, reductions in CMR associated with this manipulation have been interpreted as indicating a role of perceptual fusion in the phenomenon. To determine whether the gap detection advantage associated with comodulated flanking bands shows a similar sensitivity to gating asynchronies among components, this experiment tested for the effect of an asynchrony between the signal band and the flanking bands. The second stimulus manipulation was that of embedding the gated 'core' of comodulated bands within an ongoing stream of random bands. Importantly, the random bands in this temporal surround were in all respects the same as the comodulated bands in terms of frequency, bandwidth and level, but differed in that the envelope patterns were independent across bands (see below, Fig. 3 ). The use of such a random temporal surround, or fringe, as an acoustic context for comodulated noise bands has been used in the characterization of CMR by Grose et al. (2005b). They demonstrated that the magnitude of masking release for a tone due to the presence of comodulated flanking bands could be markedly reduced if the comodulated bands were embedded in a random temporal fringe. One way to view this finding is that the random temporal fringe diminishes the weight placed upon the regularity of the spectro-temporal coherence occurring during the listening intervals; i.e., spectro-temporal irregularities outside the listening intervals reduce the weight placed on across-channel cues, and this reduction persists into the listening intervals. The present experiment incorporated a similar temporal fringe within the context of a gap detection paradigm. A similar behavior of the gap detection advantage relative to CMR in this manipulation would be consistent with a common underlying mechanism.

\section{A. Method}

The same waveform library from experiment 1 was used in experiment 2 . For gated conditions, the stimuli were presented only during the 500 -ms observation intervals. The stimuli were gated on and off for the intervals using raised-cosine ramps, $50 \mathrm{~ms}$ in duration. The same six observers participated in experiment 2. For each noise type (GN and SN), nine corresponding conditions were constructed. Five of these were similar to the continuous conditions of 
experiment 1; that is, gap detection for gated stimuli was measured for the signal band alone, and then in the presence of either one or four synchronously gated flanking bands. These flanking bands were either comodulated with the signal band or were independent. The next three conditions were designed to probe the mechanisms underlying the detection advantage observed in experiment 1 . The first of these conditions tested for asynchrony effects by presenting the $1000-\mathrm{Hz}$ signal band continuously but gating on the comodulated flanking bands only during the observation intervals. The other two conditions tested the effect of a temporal fringe by embedding the core comodulated complex consisting of the signal and four flanking bands into a temporal fringe consisting of five noise bands having the same center frequencies and bandwidths as the core bands. In one condition these fringe bands were also comodulated with respect to each other; in the other condition they were statistically similar to the core bands but were independent. This is illustrated in Fig. 3 for three noise bands, showing the comodulated core surrounded by the random fringe. The fringe bands were presented continuously except during the observation intervals that contained the core comodulated bands. The transitions from the temporal fringe to the observation intervals were accomplished by means of overlapping 50-ms ramps that rendered the transitions perceptually seamless; i.e., as the temporal fringe was gated off at the start of an observation interval, the core comodulated bands were gated on, and vice versa at the end of an observation interval. The condition where the temporal fringe consisted of five comodulated bands therefore reduced to one of continuous comodulated noise presentation. The final condition was a retest of the continuous baseline condition of experiment 1 (signal band alone). In the rare event that an observer could not generate a reliable threshold below the ceiling value of $499 \mathrm{~ms}$ in a particular threshold estimation track, an estimate of $499 \mathrm{~ms}$ was entered for that track. This occurred in two conditions for a total of three tracks for Observer 5.

\section{B. Results and Discussion}

The results of the five gated conditions that complement the continuous conditions of experiment 1 are shown in Fig. 4 for each noise type. Two key data patterns are evident: [1] unlike experiment 1 , no gap detection advantage is evident in the presence of comodulated flanking bands; and [2] there is some indication that gap detection performance is better with comodulated flanking bands than random flanking bands. Before examining these key patterns more quantitatively, a preliminary question concerns the effect of gating on gap detection performance in the baseline single band alone condition. Comparing the upper two panels of Fig. 2 to the corresponding panels of Fig. 4, there is a trend for the baseline thresholds to be lower in the gated conditions than in the continuous conditions. To test this, a two-factor RANOVA was undertaken with factors mode of presentation (gated vs. continuous) and noise type (GN vs. SN). Note that the data for the continuous mode of presentation used in this analysis were those collected as part of experiment 2 , so that all data in the analysis came from the same testing sessions. ${ }^{1}$ The analysis indicated a significant effect of noise type $\left(\mathrm{F}_{1,5}=\right.$ 72.47; $\mathrm{p}<0.01)$, but no effect of presentation mode $\left(\mathrm{F}_{1,5}=4.27 ; \mathrm{p}=0.09\right)$ and no interaction between these two factors.

To determine the effect of flanking band presence on gap detection thresholds, separate RANOVAs were performed on the GN and SN datasets. For the GN stimuli, there was a significant effect of condition $\left(\mathrm{F}_{4,20}=4.21 ; \mathrm{p}=0.01\right)$. Post-hoc contrasts indicated that the presence of a single flanking band - either comodulated or random - raised thresholds significantly above baseline $\left(F_{1,5}=7.48 ; p=0.04\right.$ and $F_{1,5}=9.16 ; p=0.03$, respectively $)$, as did the presence of four random flanking bands $\left(F_{1,5}=7.64 ; p=0.04\right)$. The presence of four comodulated flanking bands did not shift threshold from baseline $\left(\mathrm{F}_{1,5}=1.99 ; \mathrm{p}=0.22\right)$. To

${ }^{1}$ For the baseline continuous conditions that were replicated between experiments 1 and 2 , the group mean threshold for the GN band was $67 \mathrm{~ms}$ in experiment 1 and $63 \mathrm{~ms}$ in experiment 2. For the SN band, the corresponding thresholds were $111 \mathrm{~ms}$ and $97 \mathrm{~ms}$, respectively. 
determine the separate effects of number of flanking bands ( 1 or 4 ) and modulation type (comodulated or random), the four conditions with flanking bands were submitted to a separate RANOVA. The results indicated no significant main effect of either factor $\left(\mathrm{F}_{1,5}=0.90 ; \mathrm{p}=\right.$ 0.39 and $\mathrm{F}_{1,5}=3.18 ; \mathrm{p}=0.13$, respectively), but the interaction between them was significant $(\mathrm{F} 1,5=11.28 ; \mathrm{p}=0.02)$. The interaction was due to thresholds being equivalent for the case of a single flanking band - comodulated or random - but thresholds being higher with four random flanking bands than with four comodulated bands. For the GN stimuli, therefore, thresholds were generally elevated relative to baseline by the introduction of flanking bands, particularly four random flanking bands. The presence of four comodulated flanking bands did not affect threshold appreciably.

A similar analysis of data for the $\mathrm{SN}$ stimuli revealed a slightly different pattern of results. A RANOVA indicated a significant effect of condition $\left(\mathrm{F}_{4,20}=8.62 ; \mathrm{p}<0.01\right)$, and post-hoc contrasts indicated that the presence of random flanking bands - either one or four significantly elevated threshold relative to baseline $\left(F_{1,5}=10.98 ; p=0.02\right.$ and $F_{1,5}=7.96 ; p$ $=0.04$, respectively). The presence of comodulated flanking bands had no effect on threshold. A RANOVA on the conditions with flanking bands indicated no effect of number of flanking bands $\left(\mathrm{F}_{1,5}=2.59 ; \mathrm{p}=0.68\right)$, but a significant effect of modulation type $\left(\mathrm{F}_{1,5}=16.74 ; \mathrm{p}=\right.$ 0.01 ). The interaction term was not significant. These results indicate that, for the SN stimuli, the presence of comodulated flanking bands was not beneficial for gap detection relative to baseline; however, the presence of random flanking bands resulted in poorer performance.

Overall, the pattern of results for the gated GN and SN noise types provides an unambiguous answer to the question of whether the detection advantage seen for comodulated flanking bands in experiment 1 was restricted to the continuous presentation mode. No detection advantage was measured for comodulated flanking bands gated synchronously with the signal band. The second question examined in this experiment was whether the detection advantage associated with continuous comodulated flanking bands in experiment 1 could be attributed to CMR-like mechanisms. Fig. 5 displays the results of the conditions designed to test this for each of the two noise types. The relevant reference condition in each panel is the continuous comodulated configuration (All Com.), which can be thought of as the core complex of five comodulated bands embedded in a temporal fringe consisting of the same five comodulated bands. Relative to this reference, gap thresholds appear elevated when the temporal fringe consists of either the signal band alone (Single) or the five random bands (All Ran.).

The effect of temporal asynchrony was measured by comparing thresholds in the continuous comodulated condition (All Com.) to those for the condition where the signal band was presented continuously whereas the comodulated flanking bands were gated on only for the observation intervals (Single). A RANOVA on the factors of synchrony status (All Com., Single) and noise type (GN, SN) indicated a significant effect of synchrony $\left(\mathrm{F}_{1,5}=22.92 ; \mathrm{p}<\right.$ $0.01)$ and noise type $\left(\mathrm{F}_{1,5}=10.61 ; \mathrm{p}=0.02\right)$, but no interaction between these factors. The elevation in threshold due to the introduction of temporal asynchrony for both noise types supports the interpretation that the gap detection advantage seen in the continuous comodulated condition bears some similarity to CMR.

To further test for commonalities with CMR mechanisms, a comparison was made between the two conditions where the core comodulated complex was embedded in a temporal fringe consisting either of comodulated bands (All Com.) or of random bands (All Ran.). The resulting RANOVA indicated a significant effect of temporal fringe type $\left(F_{1,5}=82.77 ; p<0.01\right)$ and a significant effect of noise type $\left(\mathrm{F}_{1,5}=16.55 ; \mathrm{p}=0.01\right)$, but no interaction between these factors. This result supports the interpretation that the gap detection advantage seen for continuous comodulated noise is sensitive to the characteristics of the temporal fringe, as is CMR for tonal signals (Grose et al., 2005b). Despite this general commonality, it should be stressed that a key 
difference between CMR for tonal signals and the gap detection advantage noted here is that, in CMR, the detection advantage is seen for both gated and continuous presentation, and is larger for continuous presentation, whereas for gaps it is observed only for continuous presentation and is absent for gated presentation.

Although the results of experiment 2 address the two specific questions that the experiment was designed to test, one aspect of the results remains puzzling. Previous experiments that have measured gap detection in a single band of noise accompanied by other bands of noise not carrying the gap have consistently shown an elevation in gap threshold due to the flanking bands and, moreover, have generally found this elevation to be more pronounced for comodulated flanking bands than for random flanking bands (Grose and Hall, 1993a; Moore et al., 1993). In contrast, the results of experiment 2 showed no consistent elevation of gap threshold in the presence of comodulated flanking bands and, overall, gap thresholds were more elevated for random flanking bands than for comodulated flanking bands. There are at least two possibilities that might account for this discrepancy. The first is that, because the observers in experiment 2 had all participated first in experiment 1 , they may have learned some cue in the continuous presentation mode of experiment 1 that they then transferred to the gated mode of experiment 2. If this cue is such that observers who do not get prior exposure to a continuous presentation mode are unlikely to learn it, then a different pattern of results may emerge from 'unexposed' observers. This might account for the different data patterns between the experiment 2 and other published studies (e.g., Grose and Hall, 1993a; Moore et al., 1993). To assess this possibility, three naive observers were recruited and tested in a subset of the gated conditions of experiment 2. These conditions were, for the GN noise type only: (1) signal band alone; (2) single comodulated flanking band; and (3) single random flanking band. The pattern of results from these three new observers was very similar to the data set of experiment 2 . That is, relative to the signal band alone baseline, thresholds were not affected by the presence of a comodulated flanking band, whereas thresholds were higher in the presence of a random flanking band than a comodulated flanking band. Transfer of a learned cue from continuous to gated presentation modes, therefore, does not appear to account for the data pattern of experiment 2.

A second possibility that might account for the difference between the results of experiment 2 and other published findings is that some of the stimulus parameters - in particular the gating characteristics of the gap - were quite different across the studies. For example, in the Grose and Hall (1993a) study, gap detection was measured in a 25-Hz wide band of (non-Gaussian) noise presented in a broad-band background noise, and gaps were imposed with 10-ms fall/ rise times. Here, the signal band was presented in quiet, with the gap imposed with a 42-ms fall/rise time. Differences across studies in the gating parameters of the imposed gap, coupled with the characteristics of the noise-band carrier, may have resulted in differences in the degree of perceptual confusion between the gap and the inherent fluctuations of the noise. As discussed earlier, it would be expected that parameter settings that minimized the perceptual confusion would result in less apparent benefit from comodulated flanking bands (cf. LFN conditions of experiment 1). In order to determine whether such stimulus characteristics underlay the performance differences, a supplementary experiment was carried out using a subset of the conditions from experiment 2 but having stimulus characteristics more similar to those of Grose and Hall (1993a).

\section{SUPPLEMENTARY EXPERIMENT}

\section{A. Method}

Observers-Eight normal-hearing observers participated, ranging in age from 19 to 49 years (mean $=32.5$ years). All had audiometric thresholds $\leq 20 \mathrm{~dB}$ HL at the octave frequencies 250 $-8000 \mathrm{~Hz}$ (ANSI, 1996). Three of the observers had participated in experiments 1 and 2; the 
remaining observers were new and were trained on the gap detection task until performance was stable.

Stimuli-As in experiments 1 and 2, the stimuli consisted of narrow bands of noise, each 25$\mathrm{Hz}$ wide. The signal band containing the gap was always the band centered at $1000 \mathrm{~Hz}$, and the remaining flanking bands, which did not contain the gap, were centered at 444, 667, 1500, and $2250 \mathrm{~Hz}$. Unlike the previous experiments, the bands were generated by a method of quadrature multiplication. For each band, two independent Gaussian noises were low-pass filtered at $12.5 \mathrm{~Hz}$ and then multiplied by a pair of tones of the same frequency but in quadrature phase. The frequency of the tone pair determined the center frequency of the resulting band. The two independent multiplied noise bands were then summed to produce the final narrow band of noise. For random bands, independent pairs of low-pass noises were used for each noise band; for comodulated bands, the same pair of low-pass noises was used for all noise bands. The signal band could be presented either alone, or in the presence of the flanking random or comodulated bands. All stimuli were gated, and were presented in a background noise consisting of a Gaussian noise low-pass filtered at $4000 \mathrm{~Hz}$ and containing spectral notches centered at each of the noisebands. The notches, designed to limit spectral splatter associated with the more abrupt gap boundaries, were one normal equivalent rectangular bandwidth $\left(E B_{N}\right)$ in width (Glasberg and Moore, 1990); for the center frequencies of 444, $667,1000,1500$, and $2250 \mathrm{~Hz}$, the notch widths were $72,94,128,182$, and $270 \mathrm{~Hz}$, respectively. The notched noise was created by means of a digital filter (TDT PD1) and presented at a level of $10 \mathrm{~dB} / \mathrm{Hz}$. The noisebands were presented at a level of $55 \mathrm{~dB} / \mathrm{band}$.

Procedure-The gap detection method was similar to that described in experiment 2. A 3AFC procedure was employed, and each observation interval was 500-ms in duration; the intervals were separated by $500 \mathrm{~ms}$. The gap was imposed on the signal band using a 10-ms raisedcosine fall/rise ramp, and gap duration was defined as the interval between the initiation of gate closure and the initiation of gate opening. The maximum allowable duration of the gap was $400 \mathrm{~ms}$. The gap was temporally centered in the target interval, which was selected at random. Each observer participated in three conditions: (1) baseline, $1000-\mathrm{Hz}$ signal band presented alone; (2) signal band plus the four comodulated flanking bands; and (3) signal band plus the four random flanking bands. At least four estimates of gap threshold - but often five, if time permitted - were measured. The observer's final threshold for each condition was taken as the geometric mean of all estimates collected for that condition. The thresholds were collected first for the single band, and then for the comodulated or random conditions. The order of these latter two conditions was randomized across observers.

\section{B. Results and Discussion}

The results of the supplementary experiment are shown in Fig. 6. Thresholds were lowest for the signal band alone and were elevated in the presence of flanking bands. Two of the observers could not reliably detect gaps below the 400-ms ceiling when flanking bands were present either comodulated or random. Such individual variability in gap detection in the presence of flanking bands is striking, but individual patterns of flanking band disruption are evident also in the results of Grose and Hall (1993a) [four observers] and Moore et al. (1993) [two observers]. The data for the six observers who generated valid thresholds for all three conditions were submitted to a RANOVA, and the analysis indicated a significant effect of condition $\left(\mathrm{F}_{2,10}=13.41 ; \mathrm{p}<0.01\right)$. Post-hoc contrasts indicated that thresholds in the presence of flanking bands were significantly elevated with respect to that for the signal band alone for both the comodulated bands $\left(F_{1,5}=6.91 ; p=0.047\right)$ and the random bands $\left(F_{1,5}=29.28 ; p=0.003\right)$. However, threshold differences between the two flanking band conditions failed to reach significance $\left(F_{1,5}=6.12 ; p=0.056\right)$. The finding that the presence of the gated comodulated flanking bands was detrimental to performance (unlike in experiment 2 ) supports the notion 
that the relative effect of comodulation depends in part on the gating characteristics of the gap. Further work is required to address how the relative effect of comodulated flanking bands depends on specific parameter settings.

\section{SUMMARY AND CONCLUSION}

The purpose of this study was to revisit earlier conclusions that gap detection in a narrow band of noise is consistently disrupted by the presence of one or more additional bands of noise that do not contain the gap. The hypothesis tested here was that this disruption is not necessarily the general case but, rather, is restricted to conditions of gated presentation. It was hypothesized that a detection advantage for gaps could be demonstrated in the presence of comodulated noise bands under conditions where the fluctuations of the noise bands were salient and, following from the characteristics of CMR, such a benefit would be most likely observed when the mode of presentation was continuous. Experiment 1 demonstrated that, indeed, detection of a gap in a continuous narrow band of noise improved in the presence of flanking bands of comodulated noise that did not contain the gap. This benefit was restricted to noise types that exhibited salient envelope fluctuations, and was not evident for low-fluctuation noise. No gap detection benefit was conferred by flanking bands of random noise. Experiment 2 indicated that no detection advantage was evident in the gated mode of presentation. It is possible that this lack of a detection advantage in the gated mode is due in part to baseline performance occurring closer to the limits of temporal acuity, per se; this possibility has the associated implication that the imposed gap is, for some reason, more perceptually distinct from the inherent fluctuations of the noise in the gated mode. Recall that there was a trend for baseline performance to be better in the gated conditions than in the continuous conditions. If thresholds are not elevated due to a confusion effect in the baseline conditions, then no detection advantage can be demonstrated, as exemplified by the LFN results of experiment 1 .

The results of experiment 2 also demonstrated that the detection advantage seen in the continuous presentation mode bore some similarity to CMR in that the advantage was sensitive to gating asynchronies across bands. Furthermore, it was sensitive to the characteristics of the temporal fringe surrounding the core comodulated bands. However, an important difference was also noted: Whereas CMR for tones can be observed in gated conditions, a detection advantage for gaps has not been observed under these conditions. This finding influences the interpretation of the effect of the random temporal fringe. In CMR, the masking release observed in the gated, comodulated condition is eliminated by the presence of the random temporal fringe; in gap detection, the random temporal fringe is detrimental to performance in that thresholds are elevated relative to those for the comodulated temporal fringe, but this does not constitute elimination of a detection advantage since there is no detection advantage in the gated, comodulated condition to begin with.

In summary, this study has demonstrated that a detection advantage for a gap carried by a single band of fluctuating noise can be conferred by the presence of comodulated flanking bands of noise that do not contain the gap. This detection advantage is observed when the noise bands are presented continuously and when performance in the baseline condition is likely limited by a confusion effect. Although it is possible that the mechanisms underlying the detection advantage are similar to those of CMR, significant differences exist between the two types of detection advantage (gap vs. tone).

\section{Acknowledgements}

We gratefully acknowledge the comments of Associate Editor Richard Freyman, Brian Moore, and two other anonymous reviewers on a previous version of this paper. This work was supported by NIDCD R01-DC01507. 


\section{References}

ANSI. American National Standards Specification for Audiometers (ANSI S3-1996). American National Standards Institute; New York: 1996.

Carlyon RP, Buus S, Florentine M. Comodulation masking release for three types of modulators as a function of modulation rate. Hear Res 1989;42:37-46. [PubMed: 2584158]

Dau, T.; Ewert, SD.; Oxenham, AJ. Effects of concurrent and sequential streaming in comodulation masking release. In: Pressnitzer, D.; de Cheveigne, A.; McAdams, S.; Collet, L., editors. Auditory Signal Processing: Physiology, Psychoacoustics, and Models. Springer Verlag; New York: 2004.

Eddins DA, Hall JW, Grose JH. The detection of temporal gaps as a function of frequency region and absolute noise bandwidth. J Acoust Soc Am 1992;91:1069-77. [PubMed: 1556308]

Glasberg BR, Moore BCJ. Derivation of auditory filter shapes from notched-noise data. Hear Res 1990;47:103-138. [PubMed: 2228789]

Glasberg BR, Moore BCJ. Effects of envelope fluctuations on gap detection. Hear Res 1992;64:81-92. [PubMed: 1490904]

Green DM, Forrest TG. Temporal gaps in noise and sinusoids. J Acoust Soc Am 1989;86:961-970. [PubMed: 2794249]

Grose JH. Gap detection in multiple narrow bands of noise as a function of spectral configuration. $\mathrm{J}$ Acoust Soc Am 1991;90:3061-3068. [PubMed: 1787244]

Grose JH, Eddins DA, Hall JW. Gap detection as a function of stimulus bandwidth with fixed highfrequency cutoff in normal-hearing and hearing-impaired listeners. J Acoust Soc Am 1989;86:174755. [PubMed: 2808923]

Grose, JH.; Hall, JW. Across-frequency processing in temporal gap detection. In: Duifhuis, H.; Wit, HP.; Horst, JP., editors. Basic Issues in Hearing. Academic Press; New York: 1988.

Grose JH, Hall JW. Gap detection in a narrow band of noise in the presence of a flanking band of noise. J Acoust Soc Am 1993a;93:1645-1648.

Grose JH, Hall JW. Comodulation masking release: Is comodulation sufficient? J Acoust Soc Am 1993b; 93:2896-2902. [PubMed: 8315153]

Grose JH, Hall JW. Cochlear hearing loss and the processing of modulation: Effects of temporal asynchrony. J Acoust Soc Am 1996;100:519-527. [PubMed: 8675845]

Grose JH, Hall JW. Multi-band detection of energy fluctuations. J Acoust Soc Am 1997;102:1088-1096. [PubMed: 9265756]

Grose JH, Hall JW, Buss E. Across-channel spectral processing. Int Rev Neurobiol 2005a;70:87-119. [PubMed: 16472632]

Grose JH, Hall JW, Buss E, Hatch DR. Detection of spectrally complex signals in comodulated maskers: effect of temporal fringe. J Acoust Soc Am 2005b;118:3774-82. [PubMed: 16419822]

Haggard MP, Hall JW, Grose JH. Comodulation masking release as a function of bandwidth and test frequency. J Acoust Soc Am 1990;88:113-118. [PubMed: 2380439]

Hall JW, Buss E, Grose JH. Spectral integration and "wideband analysis" of complex stimuli. J Acoust Soc Am. 2007accepted

Hall JW, Grose JH, Dev MB. Auditory development in complex tasks of comodulation masking release. J Speech Hear Res 1997;40:946-954.

Hall JW, Grose JH, Haggard MP. Effects of flanking band proximity, number, and modulation pattern on comodulation masking release. J Acoust Soc Am 1990;87:269-283. [PubMed: 2299038]

Hall JW, Grose JH, Joy S. Gap detection for pairs of noise bands: effects of stimulus level and frequency separation. J Acoust Soc Am 1996;99:1091-1095. [PubMed: 8609292]

Hatch DR, Arné BC, Hall JW. Comodulation masking release (CMR): Effects of gating as a function of number of flanking bands and masker bandwidth. J Acoust Soc Am 1995;97:3768-3774. [PubMed: 7790654]

Kohlrausch A, Fassel R, van der Heijden M, Kortekaas S, van de Par S, Oxenham AJ. Detection of tones in low-noise noise: Further evidence for the role of envelope fluctuations. Acustica 1997;83:659669. 
Moore BCJ, Jorasz U. Detection of changes in modulation depth of a target sound in the presence of other modulated sounds. J Acoust Soc Am 1992;91:1051-1061.

Moore BCJ, Shailer MJ, Black MJ. Dichotic interference effects in gap detection. J Acoust Soc Am 1993;93:2130-2133. [PubMed: 8473626]

Rice, SO. Mathematical analysis of random noise. In: Wax, N., editor. Selected Papers on Noise and Stochastic Processes. Dover; New York: 1954.

Schooneveldt GP, Moore BCJ. Comodulation masking release (CMR) as a function of masker bandwidth, modulator bandwidth and signal duration. J Acoust Soc Am 1989a;85:273-281. [PubMed: 2921409]

Schooneveldt GP, Moore BCJ. Comodulation masking release (CMR) for various monaural and binaural combinations of the signal, on-frequency, and flanking bands. J Acoust Soc Am 1989b;85:262-272. [PubMed: 2921408]

Shailer MJ, Moore BCJ. Gap detection as a function of frequency, bandwidth and level. J Acoust Soc Am 1983;74:467-473. [PubMed: 6619424]

Viemeister NF, Bacon SP. Forward masking by enhanced components in harmonic complexes. J Acoust Soc Am 1982;71:1502-1507. [PubMed: 7108024]

Yost WA, Sheft S. Across-critical-band processing of amplitude-modulated tones. J Acoust Soc Am 1989;85:848-857. [PubMed: 2925999] 

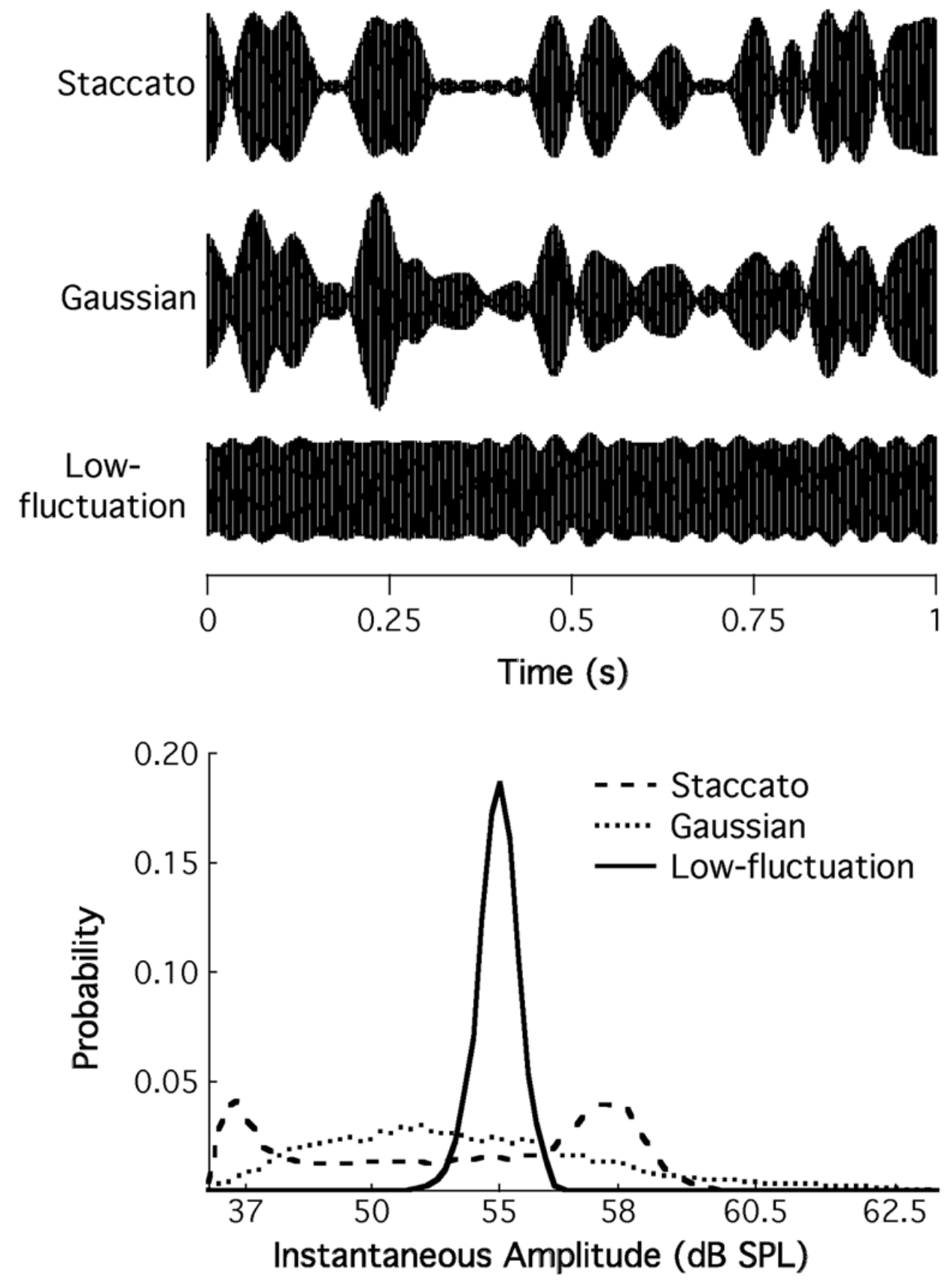

Fig. 1.

Upper panel: Sample waveforms of the three types of $25-\mathrm{Hz}$ wide noise bands. Lower panel: Distribution of instantaneous amplitudes for the three noise types. Relative to the Gaussian noise band, with its Raleigh envelope distribution, the staccato noise band was processed to accentuate the interrupted quality of the noise, giving it a bimodal envelope distribution. The low-fluctuation noise band was processed to minimize envelope fluctuations, giving it a narrow envelope distribution centered at $55 \mathrm{~dB}$ SPL. See text for stimulus generation procedure. 


\section{Continuous Noise}



Fig. 2.

Gap detection thresholds as a function of the number of flanking bands for continuous stimulus presentation. Data are shown in separate panels for each type of noise (SN, GN and LFN), with the group means overlaid in the lower right panel (circles: SN, squares: GN, triangles, LFN). In each panel group means are shown by large symbols, \pm 1 standard deviation, with filled symbols indicating comodulated conditions and open symbols indicating random conditions. Individual data points are shown clustered to the side of the respective mean in each panel. 


\section{Leading Random Comodulated Trailing Random} Fringe Interval

Fringe

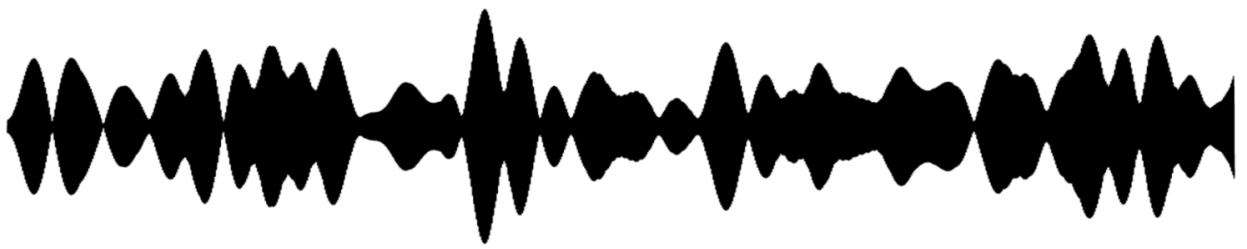

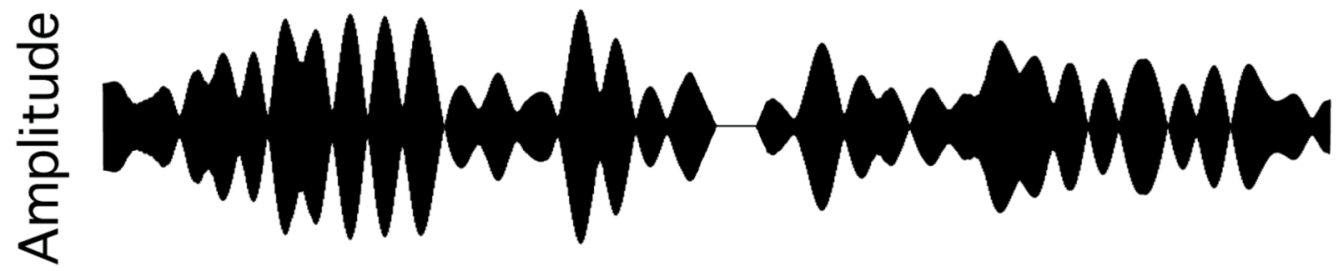

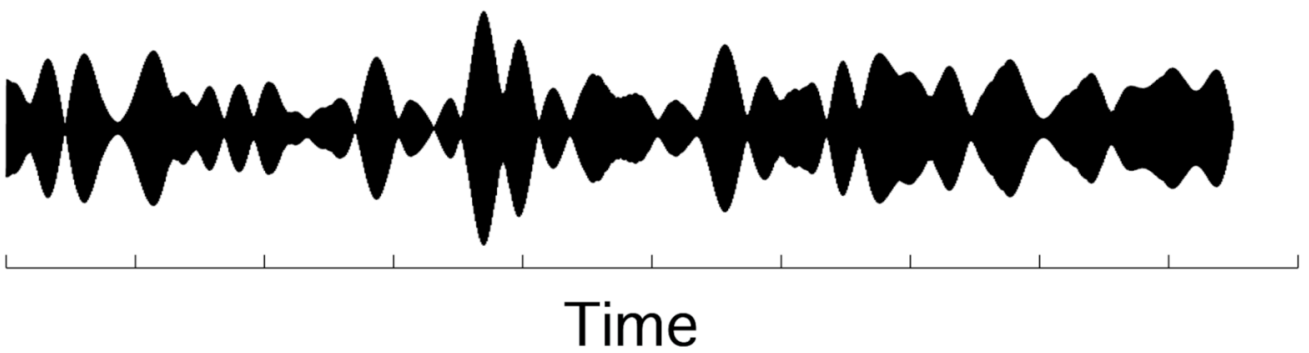

Fig. 3.

Sample waveforms of 3 noise bands showing random temporal fringe surrounding a comodulated core. The gap is carried only by the center band. 


\section{Gated Noise}

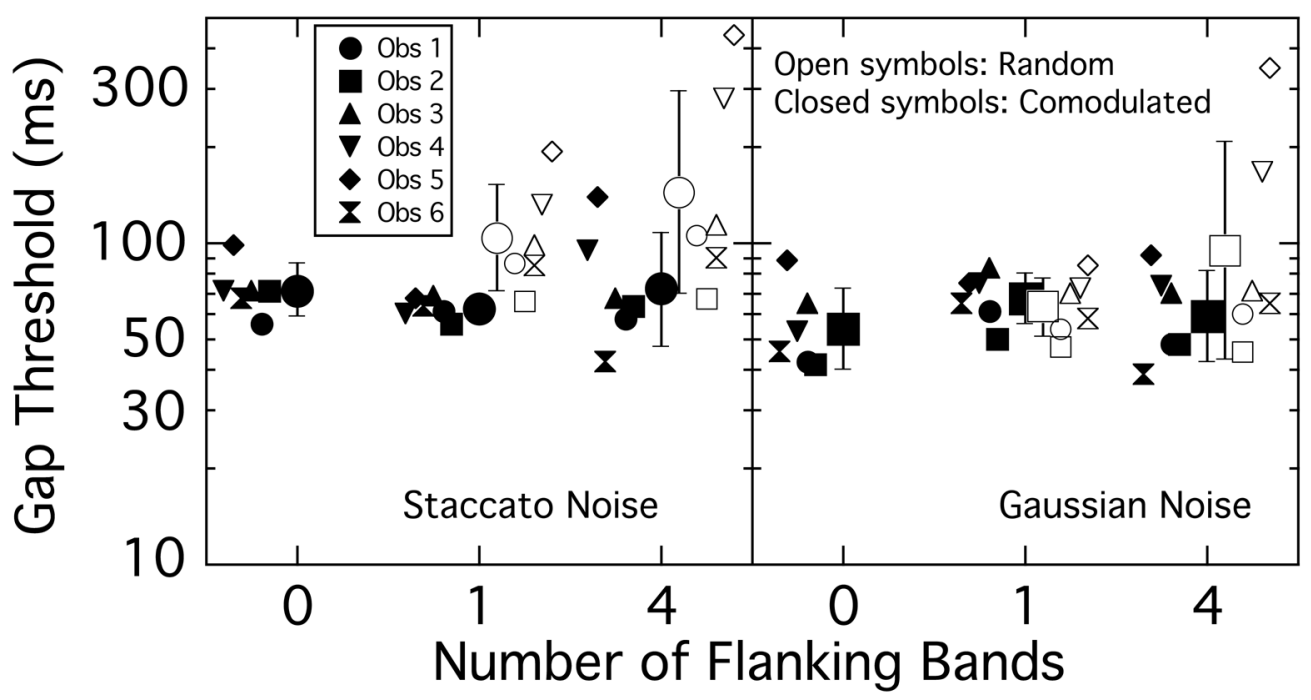

Fig. 4.

Gap detection thresholds as a function of the number of flanking bands for gated noise. Data are shown in separate panels for staccato noise and Gaussian noise. Otherwise, as Fig. 2. 


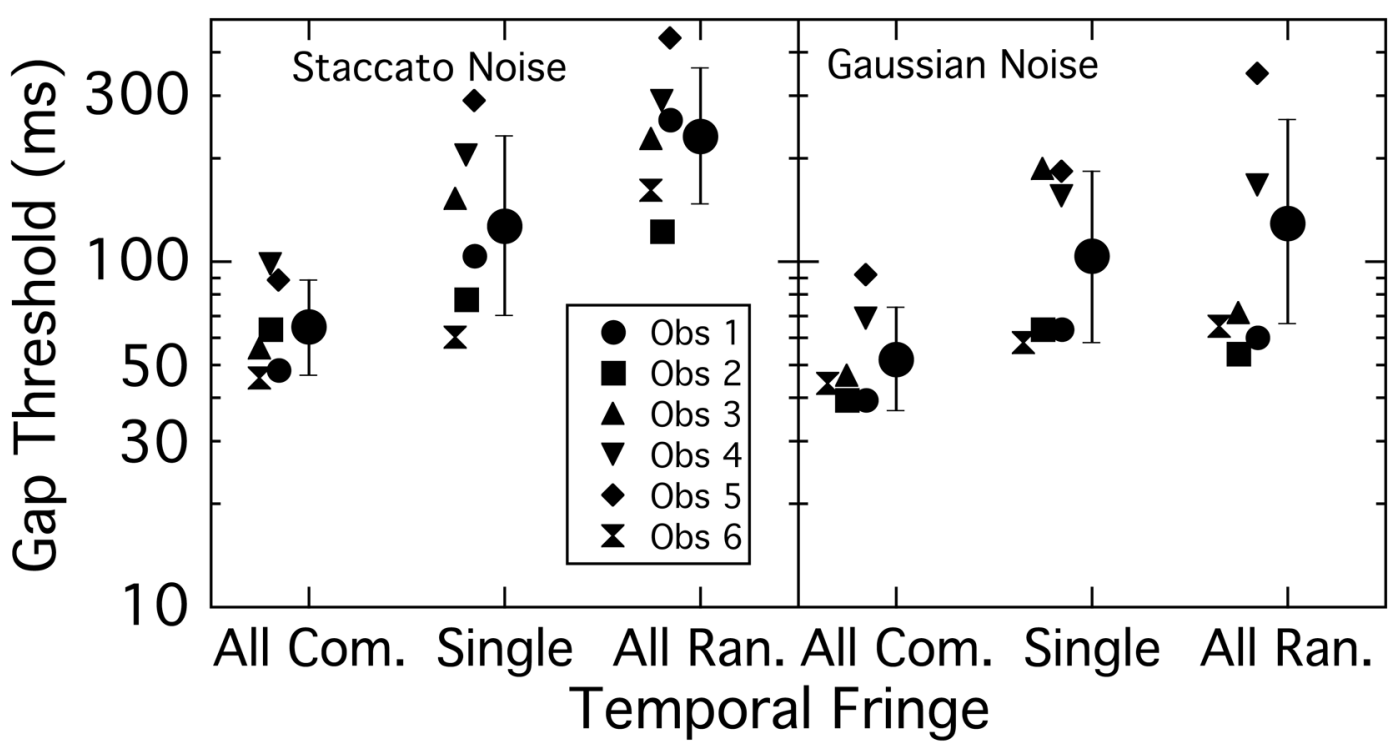

Fig. 5.

Gap detection thresholds as a function of the characteristics of the temporal fringe. The core, consisting of the synchronously gated signal band and four comodulated flanking bands, had a temporal fringe of either the same five comodulated bands (All Com.), the signal band alone (Single), or five random bands (All Ran.). Data are shown in separate panels for staccato noise and Gaussian noise. In each panel group means are shown by large symbols, \pm 1 standard deviation, with individual data points clustered to the side of the respective mean. 


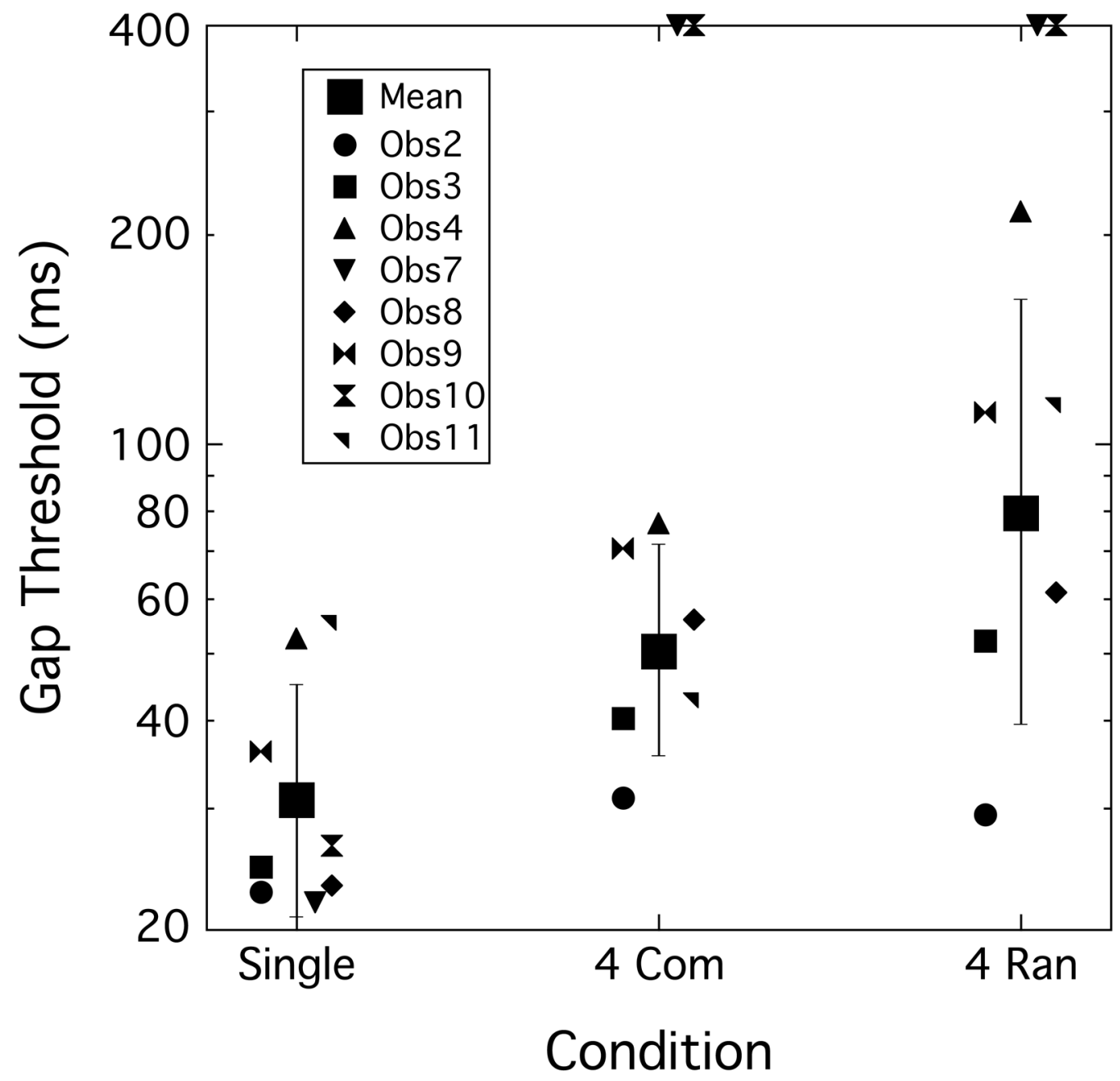

Fig. 6.

Gap detection thresholds as a function of the presence and type of flanking bands: Single - no flanking bands present; 4 Com - four comodulated flanking bands; 4 Ran - four random flanking bands. Group means are shown by large squares, \pm 1 standard deviation, with individual data points clustered around the respective mean. Symbols on the top edge of the graph indicate ceiling performance at $400 \mathrm{~ms}$. 